\title{
RELAÇÃO ENTRE DESIGUALDADE DE RENDA E CRESCIMENTO ECONÔMICO NOS MUNICÍPIOS BRASILEIROS: O QUE MUDOU NA DÉCADA \\ 2000-2010?
}

RELATIONSHIP BETWEEN INCOME INEQUALITY AND ECONOMIC GROWTH IN BRAZILIAN MUNICIPALITIES: WHAT HAS CHANGED IN THE DECADE

2000-2010?

\section{RELACIÓN ENTRE LA DESIGUALDAD DE INGRESOS Y EL CRECIMIENTO ECONÓMICO EM LOS MUNICIPIOS BRASILEÑOS: ¿QUÉ HA CAMBIADO EN \\ LA DÉCADA 2000-2010?}

Rosiane Tapias Rodrigues

Mestre em Ciências Contábeis pela Fundação

Instituto Capixaba de Pesquisa em

Contabilidade, Economia e Finanças

(FUCAPE), Brasil

rosetapias@ hotmail.com

\section{Bruno Funchal}

Pós-doutor em Economia pelo Instituto

Nacional de Matemática Pura e Aplicada

(IMPA), Brasil; Professor Titular da FUCAPE

bfunchal@fucape.br

\section{Victor Rangel dos Santos Rodrigues}

Graduando em Ciências Econômicas pela

FUCAPE

victorrangelrodrigues@gmail.com

\section{Danilo Soares Monte-Mor}

Doutor em Ciências Contábeis e

Administração pela FUCAPE; Professor

Assistente da FUCAPE

danilo@fucape.br
Contextus

ISSNe 2178-9258

Organização: Comitê Científico Interinstitucional Editor Científico: Carlos Adriano Santos Gomes

Avaliação : Double Blind Review pelo SEER/OJS

Revisão: Gramatical, normativa e de formatação

Recebido em 03/08/2016

Aceito em 14/11/2016

$2^{\mathrm{a}}$ versão aceita em $23 / 12 / 2016$

\section{RESUMO}

Este artigo buscou verificar a validade da hipótese de Kuznets para os municípios brasileiros nos anos de 1991, 2000 e 2010. Kuznets (1955) sugere que o crescimento econômico em seu estágio inicial vem acompanhado com o aumento da desigualdade de renda, atingindo um ponto de máximo e, posteriormente, diminui na medida em que a economia se desenvolve, em um padrão gráfico de "U" invertido. O estudo investiga tal hipótese por meio da aplicação de dados em painel, utilizando informações do Atlas do Desenvolvimento Humano do Brasil (PNDU, 2013), usando os coeficientes de GINI e L de Theil como medidas de desigualdade e a renda per capta como medida de crescimento. Os resultados obtidos indicam que a hipótese de Kuznets é aceita sob algumas particularizações, porém é rejeitada sob a análise de um modelo mais geral.

Palavras-chave: Curva de Kuznets. Desigualdade de renda. Crescimento Econômico. Dados em Painel. Municípios Brasileiros. 


\section{ABSTRACT}

This article aimed to verify the validity of the Kuznets hypothesis for Brazilian cities in 1991, 2000 and 2010. Kuznets (1955) suggests that economic development in its early stages is accompanied with the increase in income inequality, up to a maximum point, and then decreases as the economy develops a curve in a graphic pattern of inverted " $U$ " shape. The study investigates this hypothesis by applying panel data, using information from the Atlas of Brazil Human Development (PNDU, 2013), using the Gini coefficient and Theil L as measures of inequality and per capita income as a measure of growth. The results indicate that the Kuznets hypothesis is accepted under some particularizations, but is rejected in a more general model.

Key-Words: Kuznets Curve. Income Inequality. Economic Growth. Panel Data. Brazilian Municipalities.

\section{RESUMEN}

Este artículo tiene como objetivo verificar la validez de la hipótesis de Kuznets para las ciudades brasileñas en 1991, 2000 y 2010. Kuznets (1955) sugiere que el crecimiento económico en sus primeras etapas se acompaña con el aumento de la desigualdad de ingresos, hasta un punto máximo, y luego disminuye a medida que la economía se desarrolla una curva en un patrón gráfico de forma invertida "T". El estudio investiga esta hipótesis mediante la aplicación de datos de panel, utilizando la información del Atlas de Desarrollo Humano Brasil (PNDU, 2013), utilizando el coeficiente de Gini y Theil L como medidas de la desigualdad y el ingreso per cápita como una medida del crecimiento. Los resultados indican que la hipótesis de Kuznets es aceptado en algunas particularizaciones, pero es rechazado en un modelo más general.

Palabras clave: Curva de Kuznets. Desigualdad de Ingresos. Crecimiento Económico. Datos de Panel. Municipios Brasileños.

\section{INTRODUÇÃO}

Debates acerca da relação entre concentração de renda e crescimento econômico, bem como estudos que tratam da abordagem proposta por Kuznets (1955) para essa dinâmica, estão vastamente presentes na literatura (KUZNETS, 1955; AHLUWALIA, 1976; RAM, 1989; ANAND; KANBUR, 1993; FIELDS; JAKUBSON, 1994; DAWSON， 1997; OGWANG, 2000; THORNTON, 2001; GALLUP, 2012). No Brasil, a discussão ganha relevância uma vez que elevados índices de desigualdade estão associados, entre outros, aos índices de criminalidade, educação, saúde e, por ter sido observado, nos últimos anos, um grande esforço na criação de políticas de transferência de renda, atendendo às demandas sociais por redistribuição. (MEDEIROS; BRITTO; SOARES, 2007; MARINHO; LINHARES; CAMPELO, 2011; LUPU; PONTUSSON, 2011; SACHSIDA, 2013).

O efeito da desigualdade no crescimento nem sempre é o mesmo e Kuznets (1955) argumenta que existe uma relação gráfica não linear entre as variáveis. De acordo com o autor, à medida que a economia se expandisse, a migração da mão de obra dos setores menos para os mais dinâmicos geraria crescente desigualdade 
de renda, até atingir um ponto máximo. A partir desse ponto, haveria um processo de nivelamento da produtividade da mão de obra, e a desigualdade diminuiria com o crescimento econômico (KUZNETS, 1955). A dinâmica pode ser explicada pela migração entre setores agrícolas e industriais, desenvolvimento e acessibilidade ao mercado de crédito, e o progresso técnico-científico. (ROBINSON, 1976; GREENWOOD; JOVANOVIC, 1990; GALOR; TSIDDON, 1997)

$\mathrm{Na}$ literatura dedicada a testar a proposição de Kuznets, há autores que defendem modelos lineares (WAN, 2002), porém diversos estudos empíricos utilizando dados cross-countries não rejeitam a hipótese de que a relação entre concentração e desenvolvimento assume um formato de U-Invertido (BARRO, 2008; THORTON, 2001; LIN; WENG, 2006), mas recebem críticas acerca da robustez do resultado, da qualidade e da comparação de dados heterogêneos. (BANERJEE; DUFLO, 2003; KNOWLES, 2005). De acordo com Gallup (2012), os trabalhos não acharam suporte para a hipótese quando utilizaram controle para Efeito Fixo dos países. Ademais, várias teorias tentam explicar os impactos positivos e negativos da concentração de renda, como imperfeições no mercado de crédito, custos indivisíveis de investimento, economia política, instabilidade social, taxas de poupança, fertilidade e desigualdade de renda, etc. (BARRO, 2000; CHEN, 2002; OTTER, 2009; GALLUP, 2012; CRUZ; TEIXEIRA; MONTE-MOR, 2015)

Os estudos nacionais também mostram incoerência de resultados. Enquanto alguns autores mostram-se favoráveis à hipótese do U-Invertido; têm os que são cautelosos em suas conclusões, pois seus estimadores apresentam baixo poder explicativo; e há aqueles que rejeitam a hipótese de Kuznets. Há também uma concentração artificial, manutenida por maus mecanismos governamentais, que pode influenciar nos resultados de estimativa do modelo.

(JACINTO; TEJADA, 2004; BELLUZZO; ANUATTINETO; PAZELLO, 2005; BENDER; FERNANDES, 2009; FARIAS; SANTOS; SILVA; MATOS; DE MOURA, 2010; TAQUES et al., 2011; AZEVEDO; SALGADO, 2012; LINHARES et al., 2012; MEDEIROS; SOUZA, 2013)

Como a dinâmica entre desigualdade de renda e desenvolvimento não são totalmente claras, este trabalho investiga a existência da curva de Kuznets para os municípios brasileiros, expandindo o trabalho feito por Barros e Gomes (2008). Especificamente, tem por objetivo verificar a relevância do fator temporal na observação do padrão de U-invertido. (KUZNETS, 1955; BARROS; GOMES, 2008) 
O estudo investigou a validade da hipótese para 5565 municípios brasileiros, considerando os anos de 1991, 2000 e 2010. Para tanto, foram utilizados dados do Atlas do Desenvolvimento Humano do Brasil (PNUD, 2013). Para a desigualdade foram utilizadas duas medidas (L de Theil e GINI) e para o desenvolvimento econômico a medida de renda per capita dos municípios em questão.

Não foram encontradas evidências significativas para sustentar a hipótese do U-Invertido, pois o modelo apresenta baixo poder explicativo com os coeficientes estimados pelo método de mínimos quadrados agrupados, independente da variável utilizada como medida de desigualdade, indicando que pouco da variação dos índices de desigualdade podem ser explicados pelo aumento da renda per capta. Outros estudos como os de Anand e Kambur (1993); Barros e Gomes (2007); Taques e Mazzuti apresentam resultados semelhantes. (ANAND; KAMBUR, 1993; BARROS; GOMES, 2008; TAQUES; MEZZUTI, 2011)

Este trabalho contribui para os estudos que testam a hipótese de Kuznets no Brasil, como Jacinto e Tejada (2004); Bagolin; Gabe e Ribeiro (2004); Bussman e Risco (2012); Linhares et al. (2012), etc, colocando em análise também o período entre 2000 e 2010. Os resultados encontrados indicam que a hipótese não tem capacidade para explicar a concentração de renda no país, o que abre uma lacuna para investigar outras hipóteses. (KUZNETS, 1955; JACINTO; TEJADA， 2004; BAGOLIN; GABE; RIBEIRO， 2004; BUSSMAN; RISCO, 2012; LINHARES et al., 2012)

Em termos práticos, o Brasil é um dos países mais desiguais do mundo e é pródigo em políticas ineficazes e regressivas de distribuição de renda (MARINHO et al., 2011; MEDEIROS; SOUZA, 2013), e esse quadro poderá ser revertido após o diagnóstico de como a desigualdade é formada e quais são suas consequências. Sob essa perspectiva, o objetivo deste estudo foi contribuir para a análise do problema e suas possíveis soluções.

\section{FUNDAMENTAÇÃO TEÓRICA}

\subsection{Estudos teóricos}

Os modelos utilizados para investigar as interações entre desigualdade e desenvolvimento econômico podem se dividir em três categorias: Modelos Dualistas, Modelos de Crescimento e Escolha Social. No primeiro grupo estão os modelos que analisam a interação entre desigualdade e crescimento em uma economia de dois setores, sendo um mais e 
o outro menos dinâmico. No segundo grupo estão estudos que têm como base a formalização de crescimento econômico de Solow para analisar as relações entre as duas variáveis sob a ótica das teorias de crescimento. O terceiro grupo procura estabelecer um vínculo entre desigualdade, distribuição e desenvolvimento através de trabalhos sobre economia política, teorema do eleitor mediano e políticas de transferência de renda. (JACINTO; TEJADA, 2004).

Simon Kuznets parte das indagações "A desigualdade de renda aumenta ou diminui no decorrer do crescimento econômico do país? Quais fatores determinam os níveis e tendências seculares de desigualdade de renda?" (KUZNETS, 1955) para simular, usando dados dos EUA, Inglaterra e Alemanha, migrações em um modelo de economia apenas com os setores agrícola e industrial para observar o padrão de crescimento da desigualdade até um ponto de máximo, e depois decrescer. $\mathrm{O}$ autor também introduz questões modernas como desigualdade antes e depois de impostos, relação entre desigualdade e pobreza quando há um crescimento significativo da renda média $\mathrm{e}$ as implicações da volatilidade na estimativa da desigualdade (BREEN; PEÑALOSA, 1999; LEIGHT, 2005; KANBUR, 2011; RAVALLION, 2011; BURMAN; 2012;).
Duas décadas depois, Robinson (1976) dá um tratamento mais rigoroso à hipótese, afirmando que ela "pode ser derivada de uma modelo simples e apenas com a suposição de que a economia pode ser dividida em dois setores, com diferentes distribuições de renda, e que há um aumento monotônico da população relativa de um dos setores ao longo do tempo." (ROBINSON, 1976).

Nesse mesmo ano, Ahluwalia (1976) publica um trabalho de grande relevância modelando a curva de Kuznets. Um modelo mais geral foi formulado para captar as influências e mudanças estruturais presente nos modelos dualistas. O usando dados de 40 países subdesenvolvidos, 6 socialistas e 14 desenvolvidos, dividiu a população por renda buscando identificar a relação entre as variáveis da equação 1 e as participações na renda dos grupos. O modelo amplo possui a forma:

$L_{i}=\alpha+\beta_{1} \log Y+\beta_{2} \log Y^{2}+\beta_{3} y+$
$\beta_{4} E+\beta_{5} M+\beta_{6} P+\beta_{7} A+\beta_{8} U+\beta_{9} S+$ $\varepsilon \quad(1)$

( $L_{i}=$ participação na renda total dos grupos; $Y=$ renda per capta; $y=$ taxa de crescimento do $\mathrm{PIB} ; \quad E=$ taxa de alfabetização; $M=$ taxa de matrículas na escola secundária; $P=$ taxa de crescimento pop.; $A=$ participação da agricultura no PIB; $U=$ pop. no meio urbano; $S=$ variável 
dummy) (AHLUWALIA, 1976; FARIAS et al., 2010)

Seguindo adiante na linha do tempo, chega-se em Anand e Kanbur (1993) que apresentam uma formalização do processo proposto por Kuznets. Em linhas gerais, o artigo ofereceu uma análise ampla das mudanças de distribuição de renda e população do processo, além de derivar as formas funcionais (e as condições para o ponto de inflexão) da relação entre desigualdade e desenvolvimento para seis índices de concentração de renda. (ANAND; KANBUR, 1993a, 1993b)

Barro (2000) usa uma modelagem derivada da versão estendida do modelo neoclássico de crescimento, e os resultados indicam que, além do formato de UInvertido, as relações entre desenvolvimento e desigualdade podem se diferenciar de acordo com a dinâmica e os estágios de desenvolvimento de cada economia. (BARRO, 2000)

Trabalhos mais recentes, como o de Lind e Mehlum (2010) e Huang et al. (2007), avançam na metodologia, propondo abordagens mais sofisticadas e refinadas em detrimento do modelo clássico proposto por Ahluwalia (1976), que é largamente difundido na literatura. $\mathrm{Na}$ maioria dos trabalhos que buscam identificar o formato de $\mathrm{U}$, os autores incluem no modelo um termo não linear no modelo de regressão padrão. Se esse termo for significante e o ponto de máximo estiver na base de dados, então deve existir uma relação em forma de U. Lind e Mehlum (2010) argumentam que esse critério é fraco, pois a verdadeira relação pode ser convexa, mas monotônica, e propõem um teste para aumentar a robustez do critério utilizado. (AHLUWALIA, 1976; LIND; MEHLUM, 2010).

Haung et al. (2007) usam, ao invés das tradicionais regressões propostas anteriormente, um modelo de regressão quantifica e reexaminam a validade da hipótese de Kuznets pelos diferentes quantis condicionais. Usando dados em cross-section de 75 países do estudo de Bulír, os autores acharam robustas evidências que suportam a hipótese do $\mathrm{U}$ invertido na maioria dos quantis condicionais do modelo de regressão quantílica paramétrica. Porém, para o modelo semiparamétrico, os resultados evidenciaram diferentes dinâmicas entre desigualdade e crescimento a depender se o país apresentava baixa, média ou alta desigualdade. Também acharam que as variáveis de controle têm efeitos variáveis a depender dos quantis. Segundo os autores, essas descobertas podem ajudar a explicar resultados conflitantes existentes na literatura empírica. (HUANG et al., 2007). 


\subsubsection{Efeitos positivos da desigualdade no crescimento econômico}

Para altos níveis de equidade econômica, os agentes econômicos não são incentivados a aumentar seus esforços produtivos, pois tais esforços não são acompanhados pelo aumento da renda. Como é impossível ter o controle e a observação de todos os processos produtivos, cria-se um problema de moral hazard, e o crescimento é prejudicado por perda de eficiência produtiva. (BROWNING; JOHNSON, 1984; OKUN, 2015)

A hipótese de Kaldor diz que a propensão marginal a poupar dos indivíduos aumentaria em função da renda. Sendo assim, a desigualdade seria benéfica para o crescimento econômico por concentrar renda naqueles que tem mais propensão a poupar, elevando o nível da poupança agregada e permitindo o investimento em capital físico e humano. Mas, como para a validade da hipótese a função poupança deve apresentar monotonicidade, a desigualdade exerceria efeito positivo no crescimento somente para baixa renda agregada, quando maiores níveis de concentração de renda são uma condição suficiente para algum acúmulo de capital. (ATTANASIO; BINELLI, 2003; CRUZ et al., 2015)
Dado que determinados projetos demandam um elevado investimento inicial, devido aos processos necessários para sua realização, somente indivíduos com alta renda conseguem realizá-los, por terem acesso ao mercado de crédito e captações financeiras. Esse processo tende a ter maior magnitude nos países em desenvolvimento, onde as instituições legais são menos estruturadas e o mercado de capitais é menos desenvolvido. Portanto, a desigualdade nessa ocasião viabiliza novas atividades econômicas. (BARRO, 2000).

\subsubsection{Efeitos negativos da desigualdade no} crescimento econômico

As imperfeições do mercado de crédito impactam negativamente $\mathrm{O}$ crescimento, pois excluiria a população com menor renda, já que problemas de assimetria informacional e limitações impostas por instituições legais fariam o mercado destinar recursos aos investimentos mais seguros ao invés dos mais rentáveis. (BECK et al., 2000; ZAMPROGNO et al., 2009; KIM, 2016)

Baseando-se no Teorema do Eleitor Mediano, a política econômica se preocupará em sanar as demandas do eleitorado médio que, com elevados níveis de desigualdade, possuirá menor renda, implicando em maior demanda por 
redistribuição de renda. As distorções nas alocações da economia ocasionada pela tributação necessária para financiar as políticas de transferência gerarão desestímulo ao investimento privado, ao trabalho e à poupança, impactando negativamente no crescimento. (MILANOVIC, 2000; CHO; DUGGAN, 2005)

Desigualdade de riqueza e renda geram incentivos que aumentam os níveis de violência e criminalidade, elevando os trade-offs públicos e privados, além dos gastos com saúde e segurança. Com a credibilidade das instituições afetada pela instabilidade, os riscos aumentam, diminuindo os investimentos no país. Nesse caso, as políticas redistributivas afetam positivamente o crescimento, por arrefecer a agitação sociopolítica. (ALESINA et al., 1996; KEEFER; KNACK, 2002; BALASSIANO et al., 2012)

\subsubsection{Efeito não linear da desigualdade no crescimento econômico}

Por fim, os efeitos da desigualdade no crescimento acabam por ter uma característica não linear. A dinâmica dependeria do nível do PIB per capta, tendo na acumulação de capital físico e humano os responsáveis pelo crescimento. Para menores níveis de PIB, o efeito positivo do aumento da poupança agregada compensa $o$ efeito negativo da falta de investimento em capital humano, devido ao crédito restrito, e o principal fator de crescimento é a formação bruta de capital fixo. À medida que o nível do PIB cresce, o investimento em capital humano torna-se o protagonista do desenvolvimento econômico, e os efeitos negativos - causados pelo mercado de capitais pouco desenvolvido e pela restrição de crédito - têm maior magnitude que o benefício gerado pela acumulação de capital físico. (GALOR, 2010; IRADIAN, 2005)

\subsection{Estudos nacionais}

Bêrni et al. (2002) investigaram a hipótese de Kuznets nos 333 municípios do Rio Grande do Sul em 1990. Utilizaram dados não paramétricos em cross-section para o $\mathrm{L}$ de Theil em função da renda tanto para a agropecuária quanto para a indústria e serviços. Os autores validam da hipótese de Kuznets nos municípios estudados, se utilizada a variável explicativa densidade demográfica municipal. Bagolin et al. (2004) também testaram a hipótese nos municípios do Rio Grande do Sul, e os resultados, além de não se aproximarem daquilo que foi proposto por Kuznets, apresentaram ainda um baixo poder descritivo. (BAGOLIN et al., 2004)

Jacinto e Tejada (2004) analisaram o mesmo tema para municípios do Nordeste 
brasileiro, especificamente no período de 1970 a 1991. Concluíram que os resultados obtidos não rejeitam a curva de Kuznets na relação entre concentração de renda e desenvolvimento, porém com modelos apresentando baixo poder explicativo. Ainda observam que os resultados alcançados pelo uso do método pooled e de dados em painel foram suficientes para apresentar evidências da curva do $\mathrm{U}$ invertido, seja na descrição de Ahluwalia (1976) ou na de Anand e Kambur (1993). (JACINTO; TEJADA, 2004; AHLUWALIA, $1976 ; \quad$ ANAND; KAMBUR, 1993a)

Araújo et al. (2009) utilizam dados cross-section e em painel da desigualdade em função da renda per capita para testar a hipótese de Kuznets nos 853 municípios de Minas Gerais entre 1991 e 2000. Concluem que as evidências encontradas não rejeitam o padrão de U invertido entre concentração e desenvolvimento para os municípios mineiros. (ARAÚJO et al., 2009)

Barros e Gomes (2007), para suas análises empíricas, utilizam três estimadores de painel: POLS, EF e EA para estudo dos 5.565 municípios brasileiros no período entre 1991 e 2000, com índices de Gini e L de Theil. Como conclusão, os autores destacam que as especificações utilizando o polinômio de $2^{\circ}$ grau corroboram para a hipótese de Kuznets, porém com um poder explicativo limitado.
No entanto, ao aplicar o polinômio de $3^{\circ}$ grau, não é confirmada a hipótese de Kuznets, apenas quando se usa o Gini com a variável Urbana. (BARROS; GOMES, 2008)

No trabalho de Bussmann e Risco (2012) encontra-se a investigação do tema no estado de Minas Gerais, utilizando dados do Atlas de Desenvolvimento Humano e do Censo Demográfico, e como variáveis o L de Theil, GINI e renda per capita. Através da construção da curva de Kuznets, com dados em corte transversal e em painel para os anos de 1991, 2000 e 2010, concluem que os resultados obtidos apontam para o formato de $\mathrm{U}$ tradicional em contraponto ao $\mathrm{U}$ invertido proposto por Kuznets, renunciando à proposição de que a relação entre desigualdade e crescimento é uma parábola invertida. (BUSSMANN; RISCO, 2012)

O trabalho de Penna et al. (2015) propõe uma análise mais refinada da renda, além de utilizarem o modelo de regressão quantílica e de conceitos de econometria espacial. Os autores analisaram os dados da região Nordeste, e propuseram uma decomposição vetorial da renda, entre renda do trabalho e das transferências governamentais, e seus resultados propõem que a curva de Kuznets é válida para o Nordeste, a não ser para municípios com renda extremamente concentrada; além de trazer evidências de que "as políticas 
públicas a serem implementadas com esse propósito [diminuição da desigualdade] devem ser focadas no aumento da participação da renda do trabalho", pois a geração de emprego e renda mostram ser mais eficazes no combate a desigualdade que as transferências de renda. (PENNA et al, 2015)

A inconsistência nos resultados desses trabalhos são evidências de que os modelos econométricos, dados, cobertura da amostra e período de tempo utilizado podem influenciar nos resultados da pesquisa (VOITCHOVSKY, 2005). Ainda, as especificidades de cada região podem influenciar na forma com que tais fenômenos interagem (BOURGUIGNON, 2004). A partir dessas informações, pode-se elaborar a hipótese de que o Brasil, por ser muito grande, contém regiões com características muito peculiares entre si. Essa hipótese será testada pelos procedimentos metodológicos descritos na seção "3 - Metodologia" utilizando os dados de todos os municípios brasileiros durante três décadas.

\section{METODOLOGIA}

Para que fosse possível testar a validade da hipótese de Kuznets, foram utilizados dados secundários de 5565 municípios brasileiros no período de 1990, 2000 e 2010. As informações foram retiradas do Atlas do Desenvolvimento Humano do Brasil (PNUD, 2013).

\subsection{Variáveis utilizadas}

$\mathrm{Na}$ Tabela 1 são apresentadas as variáveis utilizadas, bem como suas definições e referências.

Tabela 1- Variáveis

\begin{tabular}{l|l|l|l}
\hline \multicolumn{1}{c|}{ Variável } & \multicolumn{1}{|c|}{ Definição } & \multicolumn{1}{c}{ Proxy } & \multicolumn{1}{c}{ Referências } \\
\hline Distribuição de Renda & $\begin{array}{l}\text { Divisão das riquezas produzidas no } \\
\text { local }\end{array}$ & $\begin{array}{l}\text { Coef. GINI } \\
\text { L de Theil }\end{array}$ & $\begin{array}{l}\text { (GINI, 1921) } \\
\text { (THEIL, 1967) }\end{array}$ \\
\hline Crescimento Econômico & $\begin{array}{l}\text { Aumento da capacidade de produção de } \\
\text { bens e serviços de determinado país }\end{array}$ & $\begin{array}{l}\text { Ln da Renda } \\
\text { per Capita }\end{array}$ & (PNUD, 2013) \\
\hline
\end{tabular}

Fonte: Elaborado pelo autor.

\subsubsection{Coeficiente GINI de renda}

Mede o grau de desigualdade existente na distribuição de indivíduos segundo a renda domiciliar per capita. Quando não há desigualdade de renda, o índice assume o valor zero (0), e quando a desigualdade é máxima assume valor igual a um (1). O coeficiente GINI é dado por:

$$
G=1-\sum_{i=0}^{k-1}\left(X_{i+1}-X_{i}\right)\left(Y_{i+1}+Y_{i}\right)
$$

Na qual $G=$ Coeficiente de GINI; $X_{i}=$ Proporção acumulada da população 
até o grupo $i ; Y_{i}=$ Proporção acumulada da renda até o grupo $i$.

\subsection{2 Índice de distribuição de renda $L$ de} Theil

Mede a distribuição de renda aos indivíduos segundo a renda domiciliar per capita. Assume valor zero quando existe perfeita igualdade de renda, e tende ao infinito quando a desigualdade tende ao máximo. É dado por:

$L=\frac{1}{n} \sum_{i=1}^{n} \ln \frac{1}{n y_{i}}$

No qual $y_{i}$ é a proporção acumulada da renda até o grupo $i$

\subsubsection{Renda per capta}

Segundo a metodologia adotada pelo Atlas de Desenvolvimento Humano:

Renda per Capta $=\frac{\sum_{i=1}^{n} Y_{i}}{n}$

Sendo $Y_{i}$ a renda do indivíduo residente em domicílio particular, e $n$ o tamanho da população do munícipio. (PNUD, 2013). Valores em reais de 01/Agosto de 2010

\subsection{Estatística descritiva}

Na Tabela 2 são apresentados os coeficientes das medidas de desigualdade GINI e L de Theil e o nível de renda para os anos de 1991, 2000 e 2010.

Tabela 2 - Estatística Descritiva

\begin{tabular}{c|c|c|c|c|c|c}
\hline Medidas/ Variáveis & Ano & Média & Mínimo & Máximo & Desvio- padrão & Coeficiente de Variação \\
\hline \multirow{3}{*}{ GINI } & 1991 & 0,525 & 0,27 & 0,92 & 0,071 & 0,137 \\
& 2000 & 0,547 & 0,30 & 0,87 & 0,068 & 0,125 \\
& 2010 & 0,494 & 0,28 & 0,80 & 0,066 & 0,133 \\
\hline \multirow{3}{*}{ L de Theil } & 1991 & 0,489 & 0,12 & 3,23 & 0,153 & 0,153 \\
& 2000 & 0,514 & 0,16 & 1,78 & 0,137 & 0,267 \\
& 2010 & 0,455 & 0,14 & 1,36 & 0,130 & 0,286 \\
\hline \multirow{3}{*}{ Renda } & 1991 & 243,83 & 33,24 & 1185,28 & 143,57 & 0,611 \\
& 2000 & 338,54 & 62,65 & 1759,76 & 192,44 & 0,568 \\
& 2010 & 493,60 & 96,25 & 2043,74 & 243,26 & 0,492 \\
\hline
\end{tabular}

Nota. O número de observações é igual a 16695.

Fonte: Elaboração pelo autor.

Entre 1991 e 2000, houve um aumento de $4,19 \%$ na média GINI e $5,11 \%$ na média $\mathrm{L}$ de Theil, o que indica piora na distribuição de renda. O Desvio-Padrão e o Coeficiente de Variação das variáveis de concentração de renda reduziram, indicando diminuição na dispersão da de desigualdade do período. A Renda Média aumentou R $\$ 94,71$ ou $38,84 \%$.

Entre 2000 e 2010, as médias do índice de GINI e do L de Theil diminuíram $9,69 \%$ e $11,48 \%$, respectivamente, indicando menor concentração de renda. $\mathrm{O}$ Desvio-Padrão diminuiu, indicando que a 
dispersão da desigualdade continuou arrefecendo, mas o Coeficiente de Variação das variáveis de concentração aumentou, indicando uma magnitude maior do efeito dessas variáveis comparado ao período passado. A Renda Média aqui aumentou $\mathrm{R} \$ 155,06$ ou $45,80 \%$.

O valor máximo atingido pela variável GINI foi de 0,92, em 1991 no município de Santa Helena (PB). O valor mínimo de 0,27 também ocorreu em 1991 em São Félix (TO).

No cenário agregado, nota-se uma sugestão do padrão de U-invertido, pois há aumento na desigualdade para posterior diminuição, com taxas crescentes de aumento de renda. Esta análise será aprofundada em nível municipal, objetivando captar o mesmo padrão para níveis menos agregados.

\subsection{Modelos econométricos}

O primeiro modelo utilizado para mensurar o impacto do crescimento econômico na concentração de renda nessa análise, também utilizado por Barro, é dado por:

$$
I_{i t}=\alpha_{0}+\alpha_{1} Y_{i t}+\alpha_{2} Y_{i t}^{2}+\varepsilon_{i t}
$$

Com as condições de concavidade dadas por:

$\frac{\partial I}{\partial Y_{i t}}=\alpha_{1}+2 \alpha_{2} Y_{i t}$ e $\frac{\partial^{2} I}{\partial Y_{i t}{ }^{2}}=2 \alpha_{2}$
Como condição para o padrão de UInvertido, $\alpha_{2}<0$. (BARRO, 2000)

$\mathrm{O}$ segundo modelo, que leva em conta que depois do padrão de U-Invertido a tendência da desigualdade poderia se alterar, e então utiliza um polinômio de terceiro grau, é dado por:

$$
I_{i t}=\alpha_{0}+\alpha_{1} Y_{i t}+\alpha_{2} Y_{i t}^{2}+\alpha_{3} Y_{i t}^{3}+\varepsilon_{i t}(2)
$$

Com as condições de concavidade dadas por:

$$
\begin{aligned}
& \frac{\partial I}{\partial Y_{i t}}=\alpha_{1}+2 \alpha_{2} Y_{i t}+3 \alpha_{3} Y_{i t}{ }^{2} \mathrm{e} \\
& \frac{\partial^{2} I}{\partial Y_{i t}{ }^{2}}=2 \alpha_{2}+6 \alpha_{3} Y_{i t}(2.1)
\end{aligned}
$$

Sendo que, o modelo capta a interação média entre as variáveis e, para o padrão previsto pela hipótese de Kuznets (1955):

$$
\begin{aligned}
& 2 \alpha_{2}+6 \alpha_{3} \bar{Y}<0 \quad \rightarrow \quad 6 \alpha_{3} \bar{Y}< \\
& -2 \alpha_{2} \rightarrow \quad \bar{Y}<-\alpha_{2} / 3 \alpha_{3}(2.2)
\end{aligned}
$$

Tanto para as equações (1) e (2), $I_{i t}=$ uma das medidas de desigualdade (GINI ou L de Theil) do município $i$ no tempo $t ; Y_{i t}=$ nível de renda do município $i$ no tempo $t$.

Os modelos descritos serão utilizados juntos à técnica econométrica que explora as características de dados em painel, dado por:

$I_{i t}=X_{i t} \Phi+u_{\mathrm{it}}$ 
Onde $I_{i t}=$ medida de desigualdade; $X_{i t} \Phi=$ todas as variáveis explicativas e $u_{\mathrm{it}}=$ erro (sendo que, caso o termo de erro contenha um efeito idiossincrático municipal. Se assim for, teremos $u_{\mathrm{it}}=$ $\tau_{i}+\varepsilon_{i t} ;$ com $\tau_{i}$ representando o efeito de cada município $i$, sendo constante ao longo do tempo).

Essa técnica permite duas abordagens: sob a ótica do Efeito Aleatório (EA), supomos que o termo $\tau_{i}$ não se correlaciona com os demais regressores, e sob a ótica do Efeito Fixo (EF) não assumimos essa hipótese. Assim, retira-se a média da equação (3) no tempo, subtraindoa na própria equação:

$I_{i t}-\overline{I_{i}}=\left(X_{i t}-\overline{X_{\mathrm{i}}}\right) \Phi+\varepsilon_{\mathrm{it}}-\overline{\varepsilon_{\mathrm{i}}}$

Assim, o efeito idiossincrático é eliminado, pois $\tau_{i}-\overline{\tau_{i}}=0 . "$ (BARROS;
GOMES, 2008; FÁVERO, 2013). Para estimar as equações serão usados três estimadores, sendo eles o Método dos Mínimos Quadrados Agrupados, EF e EA, depois aplicado o teste de Hausman, diagnosticando qual será o melhor (EA ou EF) para o presente estudo. Todos os modelos contêm dummies de tempo.

\section{ANÁLISE DOS RESULTADOS}

As Tabelas 3 e 4 apresentam os resultados da metodologia exposta em relação ao modelo I e II, para os estimadores de Mínimos Quadrados Agrupados (POLS) e Efeito Fixo, utilizando o índice de GINI e o L de Theil como variáveis dependentes e a renda per capta como variável independente. A discussão dos resultados vem apresentando nas subseções a seguir.

Tabela 3 - Resultado Para a Equação (1), usando a Renda

\begin{tabular}{|c|c|c|c|c|}
\hline \multirow{2}{*}{ Regressores } & \multicolumn{2}{|c|}{ Var. Dep.: Gini } & \multicolumn{2}{|c|}{ Var. Dep.: L de Theil } \\
\hline & POLS & EF & POLS & EF \\
\hline Renda $\left(\alpha_{1}\right)$ & $\begin{array}{c}-0.0899 * * * \\
(0.00700)\end{array}$ & $\begin{array}{c}0.126 * * * \\
(0.0151)\end{array}$ & $\begin{array}{c}-0.0379 * * * \\
(0.0144)\end{array}$ & $\begin{array}{c}0.486 * * * \\
(0.0324)\end{array}$ \\
\hline Renda ao quadrado $\left(\alpha_{2}\right)$ & $\begin{array}{c}0.0542 * * * \\
(0.00634)\end{array}$ & $\begin{array}{c}-0.0710 * * * \\
(0.00932)\end{array}$ & $\begin{array}{c}0.0124 * * * \\
(0.01300)\end{array}$ & $\begin{array}{c}-0.307 * * * \\
(0.0200)\end{array}$ \\
\hline Dummy de tempo $(\delta) 2000$ & $\begin{array}{c}0.0268 * * * \\
(0.00134)\end{array}$ & $\begin{array}{c}0.0139 * * * \\
(0.00145)\end{array}$ & $\begin{array}{c}0.0280 * * * \\
(0.00276)\end{array}$ & $\begin{array}{l}-0.00207 \\
(0.00310)\end{array}$ \\
\hline Dummy de tempo $(\delta) 2010$ & $\begin{array}{c}-0.0201 * * * \\
(0.00149)\end{array}$ & $\begin{array}{c}-0.0476 * * * \\
(0.00247)\end{array}$ & $\begin{array}{c}-0.0269 * * * \\
(0.00306)\end{array}$ & $\begin{array}{c}-0.0899 * * * \\
(0.00528)\end{array}$ \\
\hline Constante $\left(\alpha_{0}\right)$ & $\begin{array}{l}0.542 * * * \\
(0.00152)\end{array}$ & $\begin{array}{l}0.501 * * * \\
(0.00303)\end{array}$ & $\begin{array}{l}0.497 * * * \\
(0.00313)\end{array}$ & $\begin{array}{l}0.399 * * * \\
(0.00649)\end{array}$ \\
\hline Condição de concavidade é atendida? & NÃO & SIM & NÃO & SIM \\
\hline $\mathbf{N}^{0}$ de observações & 16695 & 16695 & 16695 & 16695 \\
\hline$R^{2}$ & 0.102 & 0.188 & 0.030 & 0.077 \\
\hline
\end{tabular}

Notas. O número de observações é igual a 5.565 para 1991, 2000, 2010. Renda é a renda per capita em R\$ 1000.

A estatística t está apresentada entre parênteses. O R2 apresentado é o R2 whitin.

$\mathrm{O}$ teste de Hausman rejeita o modelo de EA no caso de Gini e do L de Theil. (entre parênteses o p-valor).

$(* * * \mathrm{P}<0.01, * * \mathrm{P}<0.05, * \mathrm{P}<0.1)$

Fonte: Elaborado pelo autor. 
Tabela 3 - Resultado Para a Equação (1), usando a Renda

\begin{tabular}{|c|c|c|c|c|}
\hline \multirow{2}{*}{ Regressores } & \multicolumn{2}{|c|}{ Var. Dep.: Gini } & \multicolumn{2}{|c|}{ Var. Dep.: L de Theil } \\
\hline & POLS & EF & POLS & EF \\
\hline Renda $\left(\alpha_{1}\right)$ & $\begin{array}{c}0.0694 * * * \\
(0.0142)\end{array}$ & $\begin{array}{c}0.541 * * * \\
(0.0234)\end{array}$ & $\begin{array}{l}0.452 * * * \\
(0.0291)\end{array}$ & $\begin{array}{l}1.623 * * * \\
(0.0493)\end{array}$ \\
\hline Renda ao quadrado $\left(\alpha_{2}\right)$ & $\begin{array}{c}-0.244 * * * \\
(0.0241) \\
\end{array}$ & $\begin{array}{c}-0.690 * * * \\
(0.0285)\end{array}$ & $\begin{array}{c}-0.906^{* * * *} \\
(0.0493) \\
\end{array}$ & $\begin{array}{c}-2.004 * * * \\
(0.0601)\end{array}$ \\
\hline Renda ao cubo $\left(\alpha_{3}\right)$ & $\begin{array}{c}0.144 * * * \\
(0.0113)\end{array}$ & $\begin{array}{c}0.283 * * * \\
(0.0124)\end{array}$ & $\begin{array}{c}0.444 * * * \\
(0.0230)\end{array}$ & $\begin{array}{l}0.777 * * * \\
(0.0261)\end{array}$ \\
\hline Dummy de tempo $(\delta) 2000$ & $\begin{array}{c}0.0253 * * * \\
(0.00134)\end{array}$ & $\begin{array}{l}0.00280 * \\
(0.00149)\end{array}$ & $\begin{array}{c}0.0232 * * * \\
(0.00274)\end{array}$ & $\begin{array}{c}-0.0326 * * * \\
(0.00316)\end{array}$ \\
\hline Dummy de tempo $(\delta) 2010$ & $\begin{array}{c}-0.0210 * * * \\
(0.00149)\end{array}$ & $\begin{array}{c}-0.0683 * * * \\
(0.00257)\end{array}$ & $\begin{array}{c}-0.0297 * * * \\
(0.00303)\end{array}$ & $\begin{array}{l}-0.147 * * * \\
(0.00543) \\
\end{array}$ \\
\hline Constante $\left(\alpha_{0}\right)$ & $\begin{array}{c}0.523 * * * \\
(0.00214) \\
\end{array}$ & $\begin{array}{c}0.442 * * * \\
(0.00393) \\
\end{array}$ & $\begin{array}{c}0.438 * * * \\
(0.00436) \\
\end{array}$ & $\begin{array}{c}0.236 * * * \\
(0.00831) \\
\end{array}$ \\
\hline Condição de concavidade é atendida? & NÃO & SIM & NÃO & SIM \\
\hline $\mathbf{N}^{0}$ de observações & 16695 & 16695 & 16695 & 16695 \\
\hline$R^{2}$ & 0.111 & 0.225 & 0.051 & 0.145 \\
\hline
\end{tabular}

Notas. O número de observações é igual a 5.565 para 1991, 2000, 2010. Renda é a renda per capita em R\$ 1000.

A estatística t está apresentada entre parênteses. O R2 apresentado é o R2 whitin.

O teste de Hausman rejeita o modelo de EA no caso de Gini e do L de Theil. (entre parênteses o p-valor).

$(* * * \mathrm{P}<0.01, * * \mathrm{P}<0.05, * \mathrm{P}<0.1)$

Fonte: Elaborado pelo autor.

\subsection{Equação I}

\subsubsection{Coeficiente de Gini}

Considerando a Tabela 3 e utilizando o método de mínimos quadrados agrupados (POLS) para estimação com o GINI como variável de desigualdade, foi encontrado um coeficiente positivo para renda ao quadrado $\left(\alpha_{2}>0\right.$ e significativo a $99 \%$ de confiança) e, portanto, a hipótese de Kuznets é rejeitada, pois o coeficiente estimado para $\alpha_{2}$ não satisfaz a condição de concavidade $\left(\alpha_{2}<0\right)$.

Pelo teste de Hausman rejeita-se a hipótese nula, concluindo que apenas o Efeito Fixo é consistente. Considerando o estimador de EF, o coeficiente da renda ao quadrado é negativo $\left(\alpha_{2}<0\right.$ e significativo a 99\% de confiança) e, portanto, a hipótese de Kuznets não é rejeitada, pois o coeficiente estimado para $\alpha_{2}$ satisfaz a condição de concavidade.

Como os dois estimadores apresentam resultados contrários, não é possível tirar conclusões acerca da validação da hipótese de Kuznets.

O poder explicativo do modelo é de $10,2 \%$ considerando o estimador POLS e de $18,8 \%$ considerando o estimador $\mathrm{EF}$, indicando que a variação no índice de GINI é pouco explicada pela variação da renda per capta. 


\subsubsection{Medida L de Theil}

Utilizando o método de Mínimos Quadrados Agrupados (POLS) para estimação com o L de Theil como variável de desigualdade, foi encontrado um coeficiente positivo para renda ao quadrado $\left(\alpha_{2}>0\right.$ e significativo a $99 \%$ de confiança) e, portanto, a hipótese de Kuznets é rejeitada, pois o coeficiente estimado para $\alpha_{2}$ não satisfaz a condição de concavidade $\left(\alpha_{2}<0\right)$.

$\mathrm{O}$ teste de Hausman aponta novamente para a utilização do estimador EF, e para este estimador, o coeficiente da renda ao quadrado é negativo $\left(\alpha_{2}<0\right.$ e significativo a $99 \%$ de confiança), portanto, a hipótese de Kuznets não é rejeitada, pois o coeficiente estimado para $\alpha_{2}$ satisfaz a condição de concavidade.

Novamente, os estimadores apresentam resultados opostos, não sendo possível tirar conclusões sobre a validação da hipótese utilizando o modelo I.

O poder explicativo do modelo é de $3,2 \%$ considerando o estimador POLS e de $7,7 \%$ considerando o estimador $\mathrm{EF}$, indicando que a variação na medida $\mathrm{L}$ de Theil é pouco explicada pela variação da renda per capta.

\subsection{Equação II}

Considerando a Tabela 4, independente da variável utilizada como medida de desigualdade (GINI ou L de Theil), quando o modelo é estimado a partir do método de Mínimos Quadrados Agrupados (POLS), a condição de concavidade dada por $\bar{Y}<-\alpha_{2} / 3 \alpha_{3}$ não é satisfeita para todos os municípios.

Pelo teste de Hausman rejeita-se a hipótese nula concluindo que apenas o Efeito Fixo é consistente neste caso e, do mesmo modo, independente da medida de desigualdade utilizada, a condição de concavidade do modelo II não é atendida para todos os municípios, rejeitando a Hipótese de Kuznets para o modelo II.

O poder explicativo do modelo para o estimador POLS e índice de GINI é de $11,1 \%$ e para medida $L$ de Theil é de $22,5 \%$, e considerando o estimador EF e índice GINI é de $5,17 \%$ e para a medida L de Theil é de $14,5 \%$. Em todos os casos, o resultado indica que a variação nas medidas de distribuição de renda é pouco explicada pela variação da renda per capta.

De forma geral, os resultados do modelo I sugerem sensibilidade do modelo ao estimador utilizado, e os resultados do modelo II rejeitaram a hipótese de Kuznets. O estudo conflui com os resultados obtidos por Anand e Kambur (1993); Barros e Gomes (2008), Taques e Mezzuti (2011); Gallup (2012), que não encontram evidências do padrão de U-invertido, e conflui com o estudo de Jacinto e Tejada (2004) na análise de poder explicativo da 
renda per capta na variação das medidas de distribuição de renda. (ANAND; KAMBUR, 1993; JACINTO; TEJADA, 2004; BARROS; GOMES, 2008; TAQUES; MEZZUTI, 2011; GALLUP, 2012)

\section{CONCLUSÕES}

O objetivo deste trabalho foi verificar se a hipótese de Kuznets é válida para 5567 municípios brasileiros nos anos de 1991, 2000 e 2010, expandindo o trabalho feito por Barros e Gomes (2008). A hipótese propõe que a concentração de renda de determinada economia cresce no curto prazo com o crescimento econômico, até chegar a um ponto de máximo para depois decrescer. (BARROS; GOMES, 2008)

Utilizando um modelo com polinômio de $2^{\circ}$ grau, os resultados divergem. Quando utilizado o estimador de Efeito Fixo, o coeficiente da Renda ao quadrado achado é negativo (-0.0710 para GINI e -0.307 para L de Theil), sugerindo que com o crescimento de renda, os níveis de desigualdade aumentam, chegam a um ponto máximo e diminuem, em um formato gráfico de U-Invertido. Porém, os resultados obtidos através do estimador POLS sugerem o oposto, pois o coeficiente da Renda ao quadrado neste caso é positivo (0.0542 para GINI e 0.0124 para L de
Theil). Sob as circunstâncias metodológicas deste estudo, o estimador EF mostrou-se enviesado para baixo, enquanto o estimador POLS enviesado para cima (BOND et al., 2001), então "é esperado que uma estimação consistente do parâmetro se encontre entre os coeficientes obtidos por esses métodos". (CRUZ et al., 2015).

O primeiro modelo utilizado mostrou-se insuficiente para analisar com clareza a hipótese de Kuznets. Contudo, ao utilizar o modelo com polinômio de $3^{\circ}$ grau, que leva em consideração, além da migração do campo para a indústria, uma migração da indústria para o setor de serviços, mesmo utilizando um ferramental mais módico, os valores estimados por $\mathrm{EF}$ e POLS não atenderam às condições de concavidade da curva, logo, o padrão proposto não é satisfeito. (KUZNETS, 1955)

Analisando os dados nos períodos e as dummies de tempo, observa-se que a renda aumenta durante todos os períodos, e que os níveis de desigualdade crescem entre 1990 e 2000, período que a dummy de tempo (2000) apresenta um coeficiente positivo, e decrescem entre 2000 e 2010, período em que a dummy de tempo (2010) passa a apresentar coeficientes negativos. Ou seja, no geral, a desigualdade vem diminuindo durante a última década, 
sugerindo um aumento na renda da parcela mais pobre da população e um avanço em termos de inclusão social e diminuição da pobreza.

Os resultados deste estudo contribuem para o diagnóstico das causas dos altos índices de concentração de renda no país, e o baixo poder explicativo dos modelos indica que a variação na renda per capta não tem grande influência nos índices de desigualdade de renda no país, dado que deve ser levado em consideração no desenvolvimento ou reestruturação de políticas econômicas.

Entretanto, esse mesmo baixo poder explicativo mostra uma limitação do trabalho, caso o objetivo seja analisar o problema de desigualdade de renda de forma mais completa. Como pesquisa futura, aconselha-se a utilização de um modelo mais robusto, com mais variáveis explicativas acopladas além da renda. Tal estudo contribuirá para o entendimento da desigualdade de forma geral, além de servir como base para a elaboração de políticas públicas em geral.

\section{REFERÊNCIAS}

AHLUWALIA, M. S. Inequality, poverty and development. Journal of development economics, v. 3, n. 4, p. 307342, 1976.

ALESINA, A.; ÖZLER, S.; ROUBINI, N.; SWAGEL, P. Political instability and economic growth. Journal of Economic growth, v. 1, n. 2, p. 189-211, 1996.

ANAND, S.; KANBUR, SM R. Inequality and development: A critique. Journal of Development Economics, v. 41, n. 1, p. 19-43, 1993.

. The Kuznets process and

the inequality - development relationship. Journal of development economics, v. 40, n. 1, p. 25-52, 1993.

ARAÚJO, T. F.; FIGUEIRÊDO, L.; SALVATO, M. A. As inter-relações entre pobreza, desigualdade $\mathrm{e}$ crescimento nas mesorregiões mineiras: 1970-2000. 2009.

ATTANASIO, O.; BINELLI, C. Inequality, growth and redistributive policies. Poverty, Inequality and Growth, p. 179, 2004.

AZEVEDO, E. M.; SALGADO, P. Universidade pública deve ser grátis para quem pode pagar? Revista Brasileira de Economia, v. 66, n. 1, p. 99-116, 2012.

BAGOLIN, I. P.; GABE, J.; RIBEIRO, E. P. Crescimento e desigualdade no Rio Grande do Sul: uma revisão da curva de Kuznets para os municípios gaúchos (1970-1991). Encontro de Economia Gaúcha, v. 2, 2004.

BALASSIANO, L.; COSTA, C. M.; GOMES, F. A. Os Fatores econômicos importam? Uma análise da criminalidade no estado do Rio Grande do Sul. Fucape Business School, 2012.

BANERJEE, A. V.; DUFLO, E. Inequality and growth: What can the data say? Journal of economic growth, v. 8, n. 3, p. 267-299, 2003.

BARRO, R.J. Inequality and Growth in a Panel of Countries. Journal of economic growth, v. 5, n. 1, p. 5-32, 2000. 
Inequality and growth revisited.

ADB Working paper series on regional economic integration, 2008.

BARROS, L.C. de; GOMES, F.A.R. Desigualdade e Desenvolvimento: a hipótese de Kuznets é válida para os municípios brasileiros? Análise Econômica, v. 26, n. 50, p. 57-82, 2008.

BECK, T.; LEVINE, R.; LOAYZA, N. Finance and the Sources of Growth. Journal of financial economics, v. 58, n. 1 , p. 261-300, 2000.

BELLUZZO, W.; ANUATTI-NETO, F.; PAZELLO, E. T. Distribuição de salários e o diferencial público-privado no Brasil. Revista brasileira de economia, v. 59, n. 4, p. 511-533, 2005.

BENDER, S.; FERNANDES, R. Gastos públicos com pessoal: uma análise de emprego e salário no setor público brasileiro nos anos 90. Revista Economia, v. 10, n. 1, p. 19-47, 2009.

BOURGUIGNON, F. The poverty-growthinequality triangle. Poverty, Inequality and Growth, v. 69, 2004.

BREEN, R.; GARCIA-PEÑALOSA, C. Income inequality and macroeconomic volatility: an empirical investigation. European University Institute, 1999.

BROWNING, E. K.; JOHNSON, W. R. The trade-off between equality and efficiency. The Journal of Political Economy, p. 175-203, 1984.

BURMAN, L.E. Taxes and Inequality. Tax Law Review, v. 66, p. 563, 2012.

BUSSMANN, T. B.; RISCO, G. R. M. Mensuração da desigualdade e do crescimento no estado de Minas Gerais: estimação da curva de Kuznets. In: SEMINÁRIO SOBRE A ECONOMIA
MINEIRA, 15., 2012, Diamantina (MG). Anais... Diamantina: Cedeplar, 2012.

CHEN, Been-Lon. An inverted-U relationship between inequality and longrun growth. Economics Letters, v. 78, n. 2, p. 205-212, 2003.

CHO, S.J.; DUGGAN, John. Bargaining foundations of the median voter theorem. Journal of Economic Theory, v. 144, n. 2, p. 851-868, 2009.

CRUZ, P. B. da; TEIXEIRA, A.; MONTEMOR, D. S. O Efeito da desigualdade da distribuição de renda no crescimento econômico. Revista Brasileira de Economia, v. 69, n. 2, p. 163-186, 2015.

DAWSON, P. J. On testing Kuznets' economic growth hypothesis. Applied Economics Letters, v. 4, n. 7, p. 409-410, 1997.

FARIAS, T.A.; SANTOS, W. O.; SILVA, A. R. S.; MATOS, D. L.; DE MOURA, F. R. A teoria do U invertido: um teste empírico da hipótese de Kuznets para a relação entre o crescimento econômico e desigualdade de renda no Brasil (19762007). Revista de Economia Mackenzie, v. 8, n. $1,2010$.

FÁVERO, L. P. L. Dados em painel em contabilidade e finanças: teoria e aplicação. BBR-Brazilian Business Review, v. 10, n.1, p. 131-156, 2013.

FIELDS, G. S.; JAKUBSON, G. H. New evidence on the Kuznets curve. Cornell University. Ithaca, NY. Processed, 1994.

GALLUP, J. L. Is there a Kuznets curve. Portland State University, 2012.

GALOR, O; TSIDDON, D. Technological progress, mobility, and economic growth. The American Economic Review, p. 363-382, 1997. 
The 2008 Lawrence R. Klein

Lecture-Comparative Economic

Development: Insights from Unified

Growth Theory. International Economic

Review, v. 51, n. 1, p. 1-44, 2010.

GINI, C. Measurement of inequality of incomes. The Economic Journal, v. 31, n. 121, p. 124-126, 1921.

GREENWOOD, J.; JOVANOVIC, B. Financial Development. Growth, and the Distribution of Income, Journal of Political Economy, v. 98, 1990.

HUANG, H. C.; LIN, S. C.; SUEN, Y. B.; YEH, C. C. A Quantile Inference of the Kuznets Hypothesis. Economic

Modelling, v. 24, n. 4, p. 559-570, jul, 2007.

IRADIAN, G. Inequality, poverty, and growth: cross-country evidence. International Monetary Fund, 2005.

JACINTO, P. de A.; TEJADA, C. A. O. Desigualdade de renda e crescimento econômico nos municípios da região nordeste do Brasil: O que os dados têm a dizer. Encontro de Economia da Anpec, v. 32, 2004.

KANBUR, R. et al. Does Kuznets still matter?. Policy-Making for Indian Planning: Essays on Contemporary Issues in Honor of Montek S. Ahluwalia, Academic Foundation Press, v. 1, n. 1, p. 5-128, 2012.

KEEFER, P.; KNACK, S. Polarization, politics and property rights: Links between inequality and growth. Public Choice, v. 111, n. 1-2, p. 127-154, 2002.

KIM, J.H. A Study on the Effect of Financial Inclusion on the Relationship Between Income Inequality and Economic Growth. Emerging Markets Finance and Trade, v. 52, n. 2, p. 498-512, 2016.
KNOWLES, S. Inequality and economic growth: the empirical relationship reconsidered in the light of comparable data. The Journal of Development Studies, v. 41, n. 1, p. 135-159, 2005.

KUZNETS, S. Economic growth and income inequality. The American economic review, v. 45, n. 1, p. 1-28, 1955.

LEIGH, A. Deriving Long-Run Inequality Series from Tax Data. Economic Record, v. 81, n. s1, p. S58-S70, 2005.

LIN, S.C.; WENG, H. W. A semiparametric partially linear investigation of the Kuznets' hypothesis. Journal of Comparative Economics, v. 34, n. 3, p. 634-647, 2006.

LIND, J. T.; MEHLUM, H. With or without U? the Appropriate Test for a UShaped Relationship. Oxford bulletin of economics and statistics, v. 72, n. 1, p. 109-118, 2010.

LINHARES, F. et al. A hipótese de Kuznets e mudanças na relação entre desigualdade e crescimento de renda no Brasil. 2012.

LUPU, N.; PONTUSSON, J. The structure of inequality and the politics of redistribution. American Political Science Review, v. 105, n. 02, p. 316-336, 2011.

MARINHO, E.; LINHARES, F.; CAMPELO, G. Os programas de transferência de renda do governo impactam a pobreza no Brasil? Revista Brasileira de Economia, v. 65, n. 3, p. 267-288, 2011.

MEDEIROS, M.; BRITTO, T.; SOARES, F. Programas focalizados de transferência de renda no Brasil: contribuições para o debate. 2007. 
; SOUZA, P. Gasto público, tributos e desigualdade de renda no Brasil. 2013.

MILANOVIC, B. The median-voter hypothesis, income inequality, and income redistribution: an empirical test with the required data. European Journal of Political Economy, v. 16, n. 3, p. 367-410, 2000.

OGWANG, T. Inter-country inequality in human development indicators. Applied

Economics Letters, v. 7, n. 7, p. 443-446, 2000

OKUN, A. M. Equality and efficiency: The big tradeoff. Brookings Institution Press, 2015.

OTTER, T. Does Inequality Harm Income Mobility and Growth? An Assessment of the Growth Impact of Income and Education Inequality in Paraguay 1992-2002. Ibero-America Institute for Economic Research, 2009.

PENNA, C. M. et al. Trabalho, Transferências e Desigualdade: a Curva de Kuznets Para o Nordeste. Revista Brasileira de Estudos Regionais e Urbanos, v. 7, n. 2, p. 34-51, 2015.

PNUD - Programa das Nações Unidas para o Desenvolvimento. Atlas do desenvolvimento humano no Brasil: atlas Brasil, 2013. Disponível em: <www.atlasbrasil.org.br/2013/>.

RAM, R. Level of Development and Income Inequality: An Extension of Kuznets-Hypothesis to the World Economy. Kyklos, v. 42, n. 1, p. 73-88, 1989.

RAVALLION, M. Growth, inequality and poverty: looking beyond averages. World development, v. 29, n. 11, p. 1803-1815, 2001.
ROBINSON, S. A note on the U hypothesis relating income inequality and economic development. The American Economic Review, v. 66, n. 3, p. 437-440, 1976.

SACHSIDA, A.; DE MENDONÇA, M. J. C. Evolução e determinantes da taxa de homicídios no Brasil. Texto para Discussão, Instituto de Pesquisa Econômica Aplicada (IPEA), 2013.

TAQUES, F. H.; DA COSTA MAZZ, C. C. D. T. P. Qual a Relação entre Desigualdade de Renda e Nível de Renda Per Capita? Testando a hipótese de Kuznets para as Unidades Federativas Brasileiras. Planejamento e Políticas Públicas, v. 2, n. 35, 2011.

THEIL, H.; THEIL, H. Economics and Information Theory. 1967.

THORNTON, J. The Kuznets inverted-U hypothesis: panel data evidence from 96 countries. Applied Economics Letters, v. 8, n. 1, p. 15-16, 2001.

VOITCHOVSKY, S. Does the profile of income inequality matter for economic growth?. Journal of Economic Growth, v. 10, n. 3, p. 273-296, 2005.

WAN, G. H. Income inequality and growth in transition economies: are nonlinear models needed? WIDER Discussion Papers//World Institute for Development Economics (UNU-WIDER), 2002.

ZAMPROGNO, L. V.; DE JESUS FILHO, J.; FUNCHAL, B. Proteção ao Credor, Sistema de Informação e o Desenvolvimento do Mercado de Crédito dos Países do Mercosul. BBR-Brazilian Business Review, v. 6, n. 3, p. 312-322, 2009. 